\title{
Dome Fuji in Antarctica as a Site for Infrared and Terahertz Astronomy
}

\author{
Masumichi Seta ${ }^{1}$, Naomasa Nakai $^{1}$, Shun Ishii ${ }^{1}$, Makoto Nagai ${ }^{1}$, \\ Yusuke Miyamoto $^{1}$, Takashi Ichikawa ${ }^{2}$, Naruhisa Takato ${ }^{3}$ \\ and Hideaki Motoyama ${ }^{4}$ \\ ${ }^{1}$ Institute of Physics, University of Tsukuba, \\ 305-8571, 1-1-1 Ten-nodai, Tsukuba, Ibaraki, Japan \\ email: seta@physics.px.tsukuba.ac.jp \\ ${ }^{2}$ Astronomical Institute, Tohoku University, \\ 980-8578, Aramaki, Aoba, Sendai, Miyagi, Japan \\ ${ }^{3}$ Subaru Telescope, National Astronomical Observatory of Japan, \\ 96720, 650 North A'ohoku Place, Hilo, HI, USA \\ ${ }^{4}$ National Institute of Polar Research, \\ 190-8518, 10-3 Midoricho, Tachikawa, Tokyo, Japan
}

\begin{abstract}
Dome Fuji on the Antarctic high plateau may be a good site for terahertz astronomy because of its high altitude of $3,810 \mathrm{~m}$ and low average temperature of $-54^{\circ} \mathrm{C}$. We have demonstrated that the opacity at $220 \mathrm{GHz}$ from Dome Fuji in summer is very good and stable; $\tau=0.045 \pm 0.007$. We have developed a transportable $30 \mathrm{~cm}$ telescope to map the Milky Way in the $\mathrm{CO}(J=4-3)$ and the $[\mathrm{CI}]\left({ }^{3} P_{1}-{ }^{3} P_{0}\right)$ lines at Dome Fuji from 2014. It has a $9^{\prime}$ beam. Physical conditions such as density and temperature of molecular clouds could be derived from a direct comparison of $\mathrm{CO}(J=4-3)$ and $[\mathrm{CI}]\left({ }^{3} P_{1}-{ }^{3} P_{0}\right)$ with $\mathrm{CO}(J=1-0)$ taken by the Columbia-CfA survey. We are also developing a $1.2 \mathrm{~m}$ sub-millimeter telescope. It will be equipped with a dual superconducting device (SIS) receiver for $500 / 800 \mathrm{GHz}$. The $1.2 \mathrm{~m}$ telescope produces a $2.2^{\prime}$ beam at $492 \mathrm{GHz}$ and could map a molecular cloud entirely. It could also observe nearby galaxies in the $\mathrm{CO}(J=4-3), \mathrm{CO}(J=7-6),[\mathrm{CI}]\left({ }^{3} P_{1}-{ }^{3} P_{0}\right),[\mathrm{CI}]\left({ }^{3} P_{2}{ }^{3} P_{1}\right)$ and in continuum emission between $460-810 \mathrm{GHz}$.
\end{abstract}

Keywords. Site testing, telescopes, sub-millimeter

\section{Introduction}

Recently several detectors for sub-millimeter (Sub-mm) and terahertz (THz) astronomy have become available such as the Superconductor-Insulator-Superconductor (SIS), Hot Electron Bolometer (HEB), Microwave Kinetic Inductance Detector (MKID), in addition to the Schottky barrier diode mixer. However $\mathrm{THz}$ astronomy is challenging because of strong absorption by the Earth's atmosphere. Sub-mm and THz astronomy are only conducted at dry and high attitude site such as Mauna Kea in Hawaii and the Atacama Desert in Chile. However, the atmospheric transparency is neither low nor stable enough for sub-mm and $\mathrm{THz}$ astronomy there. Dome Fuji on the Antarctic high plateau is one of several promising sites for $\mathrm{THz}$ and infrared astronomy because of its low average temperature of $-54^{\circ} \mathrm{C}$ and high altitude of $3,810 \mathrm{~m}$. In this paper we introduce radio astronomy plan at Dome Fuji. The plan for the infrared telescope at Dome Fuji is described by Okita (2012) in these Proceedings. 


\section{Dome Fuji Station}

The Japanese Dome Fuji station is located at a latitude of $77^{\circ} 19^{\prime} \mathrm{S}$ and longitude of $39^{\circ} 42^{\prime} \mathrm{E}$ at an altitude of $3,810 \mathrm{~m}$ on the Antarctic plateau (Watanabe et al., 1999). It is about $1,000 \mathrm{~km}$ away from the Japanese Syowa station. The annual average temperature is $-54.4^{\circ} \mathrm{C}$ and the lowest temperature recorded is $-79.7^{\circ} \mathrm{C}$ (Yamanouchi et al., 2003). The maximum temperature in summer is $-21.1^{\circ} \mathrm{C}$. It is known that the average fraction of the sky obscured by clouds has been 30\% from 1995-1997 (Yamanouchi et al., 2003). The mean wind speed is $5.8 \mathrm{~m} \mathrm{~s}^{-1}$ and it rarely exceeds $10 \mathrm{~m} \mathrm{~s}^{-1}$. These conditions are favourable for astronomy because a high percentage of practical observational time is available in Dome Fuji.

Now the winter over station is under snow and a new Dome Fuji station is planned. Astronomy is one of key sciences for the new Dome Fuji station. The new station may be placed about $60 \mathrm{~km}$ away from the present station, where older ice and drier atmospheric conditions are expected. A high deck type of building is planned for the new station to avoid snow accumulation around the buildings. The capacity for winter is designed to be ten people for the first stage, with the possibility of future expansion. Electric power of $100 \mathrm{kVA}$ may be available. Inmarsat Satellite communication is possible from Dome Fuji. The construction hopefully will start in 2016 and be completed by 2018. Several summer traverses are planed during the construction periods, so astronomical data will be obtained soon.

\section{Opacity at Dome Fuji}

We measured atmospheric opacity at Dome Fuji from December 2006 - January 2007 (Ishii et al., 2010) and January 2010 using a tipping radiometer. We show the radiometer at Dome Fuji in Figure 1.

The instrument measured the brightness temperature of the sky at $220 \mathrm{GHz}$ with a beam of $63^{\prime}$. The scanning mirror was programmed to scan the sky every two minutes with the zenith angle between $Z=0(\sec Z=1.0)$ and $Z=70.5^{\circ}(\sec Z=3.0$ ), and the zenith opacity was derived by reducing the data for one scanning. The $220 \mathrm{GHz}$ opacity is an important parameter needed to evaluate the site because it has been measured

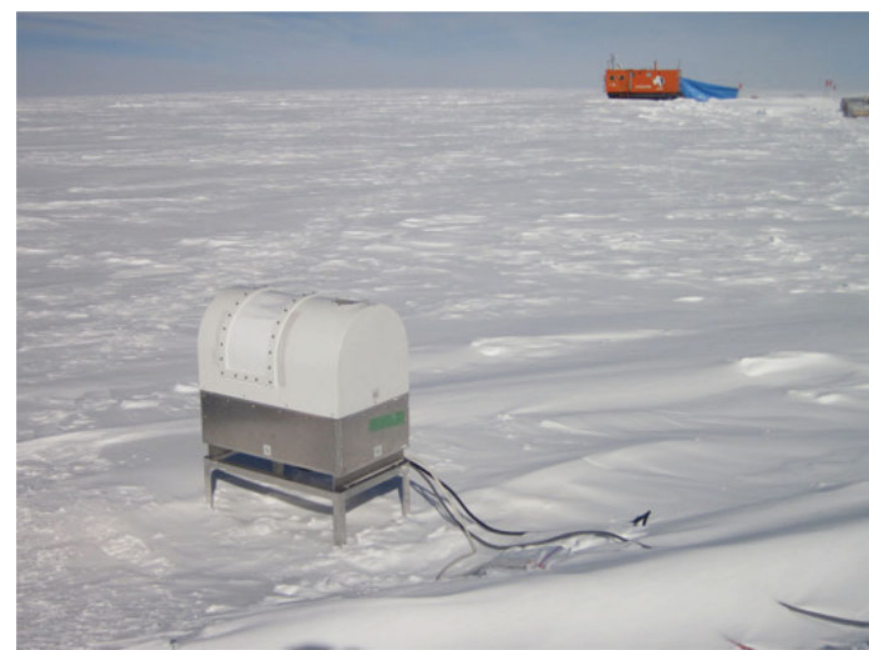

Figure 1. Radiometer at Dome Fuji in 2010. 
at various sites in the world. We show the results in Figure 2. The opacity was low and stable; $\tau=0.045 \pm 0.007$ for about one month in summer from December 2006 to January 2007. The low and stable value of 0.054 was again obtained for about one week in the summer of January 2010. This low value of the opacity is comparable with that of the best season in the Atacama Desert in Chile.

Computer simulations show the winter opacity at Dome Fuji to be much better than that of the Atacama Desert (Ishii et al., 2010). The sub-mm transparency is much improved in winter. It should noticed that new $\mathrm{THz}$ windows above $1 \mathrm{THz}$ open at Dome Fuji. Low precipitable water vapor (PWV) of $0.025 \mathrm{~mm}$ was recorded in Dome A for winter (Yang et al., 2010), and may have similar conditions to Dome Fuji. So the opacity at Dome Fuji must be very good in the winter season. We have plans to measure the opacity in winter in 2014.

\section{Route for Dome Fuji}

Dome Fuji station is about $1,000 \mathrm{~km}$ away from Syowa station. Icebreaker Shirase and snow sledges transport the most of astronomical instruments. Shirase operates only once a year. It leaves Japan in the middle of October and arrives at the coast near Syowa station in the middle of December, after picking up observers and fresh food at Fremantle in Australia. Shirase accommodates 56 boxes of 12 - $\mathrm{ft}(3.6 \mathrm{~m} \times 2.4 \mathrm{~m} \times 2.4 \mathrm{~m})$ containers. It is desirable that all instruments are designed to be packed in the 12 -ft container. A deck crane is used to transport the container from Shirase to the iced sea near Syowa station. The maximum weight of cargo on the $12-\mathrm{ft}$ container is limited to be less than $15 \mathrm{t}$. However, astronomical instruments for Dome Fuji must be designed to be much lighter than 15 t. The traverse to Dome Fuji starts from the S16 point near to Syowa. Syowa station is on a small island and all cargo for Dome Fuji is transported to S16 either by a CH-101 helicopter or by snow sledges. The maximum weight is limited to be less than $2 \mathrm{t}$ and $5 \mathrm{t}$ for the helicopter and the sledge, respectively.

It takes about 3 weeks to go from S16 to Dome Fuji. We measured the shock on the cargo during transportation from Dome Fuji to S16. The maximum shock recorded every 20 minutes is shown in Figure 3. Strong shocks were recorded at the time of lifting the instrument to/from sledge at Dome Fuji and S16. However strong shocks were also recorded from January 18 to February 2. This time corresponds to the route between middle point and the Mizuho mid-way station, where rough snow surfaces occur. New snow sledges are under development so as to reduce the strong vibration during the

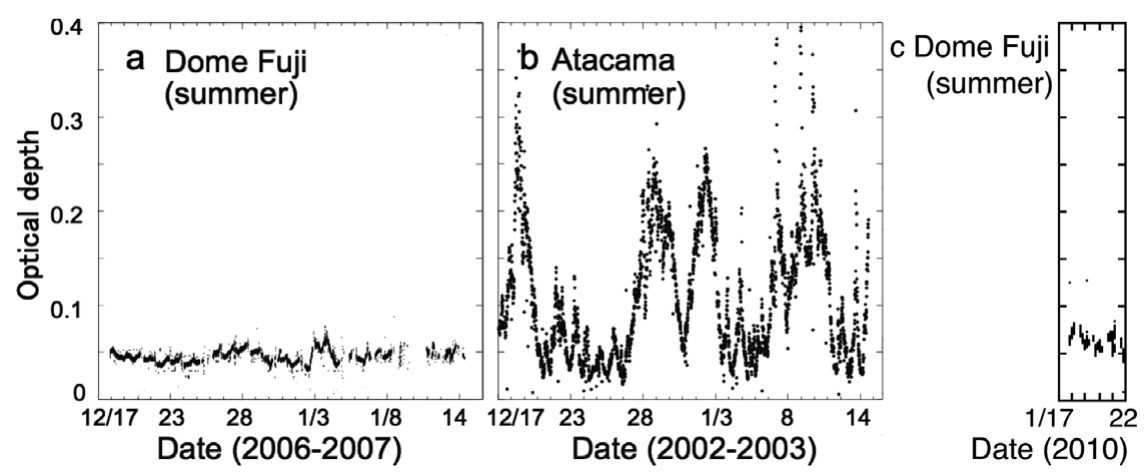

Figure 2. Zenith optical depth at $220 \mathrm{GHz}$ measured at Dome Fuji in December 2006 - January 2007: (a) January 2010; (c) comparison with the Atacama Desert in the same season; (b) data for the Atacama consisting of the best records obtained from 1995 to 2004. 
transportation on the rough snow surface. It is also possible to use a small airplane to fly to Dome Fuji directly but funds are limited for its use.

\section{The $30 \mathrm{~cm}$ Radio Telescope}

We have developed a prototype telescope for operation at Dome Fuji. It is a transportable sub-mm wave telescope (Ishii et al., 2012). It has $30 \mathrm{~cm}$ diameter offsetCassegrain antenna that produces a $9^{\prime}$ beam. It is equipped with a heterodyne SIS receiver operating at $460-492 \mathrm{GHz}$ with a quasi-optical single sideband filter. It uses a 1-GHz bandwidth spectrometer that covers a velocity width of $600 \mathrm{~km} \mathrm{~s}^{-1}$ with a velocity resolution of $0.04 \mathrm{~km} \mathrm{~s}^{-1}$.

Large scale galactic CO $(J=1-0)$ surveys revealed the distribution of molecular cloud in the 1980s (e.g., Dame et al., 1987, Scoville et al., 1987). However the physical conditions of the molecular clouds remains unclear. Multiple line observations are required to derive conditions such as density and temperature. The Univ. of Tokyo-NRO CO $(J=2-1)$ survey shows that distributions of CO $(J=2-1)$ and CO $(J=1-0)$ are similar in the Milky Way although it also shows peculiar ratios for selected regions (e.g., Sakamoto et al., 1987). The CO $(J=4-3)$ and $\mathrm{CO}(J=1-0)$ ratio may be useful to constrain the physical conditions of the molecular clouds. It is also important to have a clear picture of molecular cloud formation. The fine-structure line of neutral atomic carbon [CI] is an important tracer for phase transitions of interstellar matter from atomic to molecular. The $30 \mathrm{~cm}$ telescope is scientifically targeting survey observations in the CO $(J=4-3)$ and $[\mathrm{CI}]\left({ }^{3} P_{1}-{ }^{3} P_{0}\right)$ lines. The beam size of $9^{\prime}$ is designed to be comparable to that of Colombia-CfA CO $(J=1-0)$ survey telescope so that a direct comparison of the data will be possible.

Transportability is an important requirement so the telescope can be assembled by only a few people. The maximum weight of each component is designed to be less than $60 \mathrm{~kg}$ and the total weight is about $700 \mathrm{~kg}$. The total power consumption is also restricted to be less than $2.5 \mathrm{~kW}$, so it can be operated by small electric generators.

We have already built the telescope and tested it in Switzerland, and in northern Chile. We succeeded in mapping the Orion molecular cloud in CO $(J=4-3)$ following first light in both $\mathrm{CO}(J=4-3)$ and $[\mathrm{CI}]\left({ }^{3} P_{1}{ }^{3} P_{0}\right)$ toward Orion KL. The SSB system noise temperature including atmospheric loss was $3,000 \mathrm{~K}$ in Chile. Now we are upgrading the

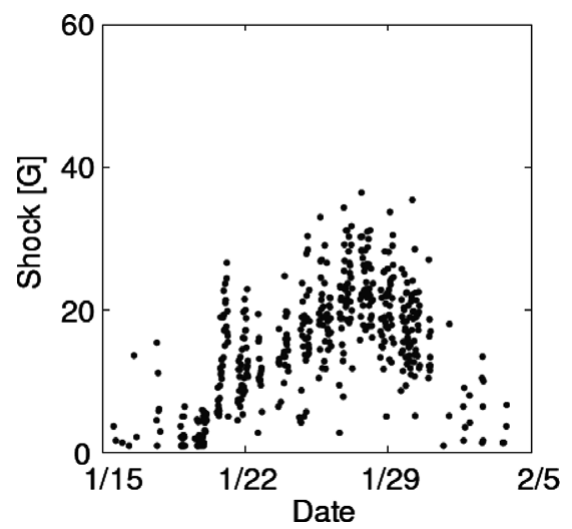

Figure 3. Maximum shock every 20 minutes during transportation from Dome Fuji to the S16 point near the Syowa station. 
SIS receiver to install a sideband separating SIS mixer and the system noise temperature is expected to be less than $500 \mathrm{~K}$ in SSB operation.

\section{Future Radio Telescopes at Dome Fuji}

We are also developing a $1.2 \mathrm{~m}$ telescope. It is a modification of the $1.2 \mathrm{~m}$ telescope used on Mt. Fuji (Sekimoto et al., 2000). The parabolic antenna produces a $2.2^{\prime}$ beam at $492 \mathrm{GHz}$. It is equipped with a $500 / 800 \mathrm{GHz}$ SIS receiver. It is possible to detect both [CI] $\left({ }^{3} P_{1}-{ }^{3} P_{0}\right)$ at $492 \mathrm{GHz}$ and $[\mathrm{CI}]\left({ }^{3} P_{2}-{ }^{3} P_{1}\right)$ at $809 \mathrm{GHz}$ simultaneously. It can also be tuned for CO $(J=4-3)$ and $\mathrm{CO}(J=7-6)$. A continuum detector is also being installed.

We will be able to reveal the structure of entire molecular clouds in both the CO and $[\mathrm{CI}]$ lines with the moderate beam size. A sub-mm continuum camera will also be installed on the $1.2 \mathrm{~m}$ telescope. We will also observe several nearby galaxies, including the Large Magellanic Cloud and the Small Magellanic Cloud. In addition, several large telescopes such as a $10 \mathrm{~m}$ class $\mathrm{THz}$ telescope are also planned.

\section{References}

Dame,T. M., Ungerechts, H., Cohen, R., de Geus, E., Grenier, I., May, J., Murphy, D., Nyman, L., \& Thaddeus, P. 1995, ApJ, 322, 706

Ishii, S., Seta, M., Nakai, N. Nagai, M., Miyagawa, N., Yamauchi,A., Motoyama, H., \& Taguchi, M. 2010, Polar Science, 3, 213

Ishii, S., Seta, M., Nakai, N., Miyamoto, Y., Nagai, M., Maezawa, H., Nagasaki, T., Miyagawa, N. Motoyama, H., Sekimoto, Y., \& Bronfman, L. 2012, $23^{\text {rd }}$ Int. Symp. Space. THz Tech.

Okita, H. 2012, IAU Symposium 288

Sakamoto, S., Hasegawa T., Handa, T., Hayashi, M., \& Oka, T. 1997, ApJ, 486, 276

Scoville, N. Z, Yun M. S., Clements, D. P., Sanders D. B., \& Waller, W. H. 2003, ApJ Suppl., 63,821

Sekimoto, Y., Yamamoto, S., Oka, T., Ikeda, M., Maezawa, H., Ito, T., Saito, G., Iwata, M., Kamegai, K., Sakai, T., Tatematsu, K., Arikawa, Y., Aso, Y., Noguchi, T., Maezawa, K., Shi, S., Saito, S., Ozeki, H., Fujiwara, H., Inatani, J., Ohishi, M., Noda, K., \& Togashi, Y. 2000, Review of Scientific Instruments, 71, 7

Watanabe, O., Kamiyama, K., Motoyama, H., Fujii, F., Shoji, H., \& Satou, K 1999, East Antarctica, Annals of Glaciology, 29, 176

Yamanouchi, T. Hirasawa, N., Hayashi, M., Takahashi S., \& Kaneto, S. 2004, Mem. Natl. Inst. Polar Res. Spec. Issue, 94

Yang, H., Kulesa, C. A., Walker, C. K., Tothill, N. F. H., Yang, J., Ashley, M. C. B., Cui, X., Feng, L., Lawrence, J. S., Luong-van, D. M, McCaughrean, M. J., Storey, J. W. V. Wang, L., Zhou, X., \& Zhu, Z. 2000, PASP, 122, 490 